05

\title{
Определение сверхвысокочастотной магнитной проницаемости альсифера из измеренной проницаемости композитных материалов
}

\author{
(C) С.Н. Старостенко ${ }^{1}$, К.Н. Розанов ${ }^{1}$, А.О. Ширяев ${ }^{1}$, А.Н. Лагарьков ${ }^{1}$, А.Н. Шалыгин ${ }^{2}$ \\ ${ }^{1}$ Институт теоретической и прикладной электродинамики РАН, \\ Москва, Россия \\ ${ }^{2}$ Московский государственный университет им. М.В. Ломоносова, \\ Москва, Россия \\ E-mail: snstar@mail.ru
}

По частотным зависимостям диэлектрической и магнитной проницаемостей композитных материалов, состоящих из порошка альсифера (сплава $\mathrm{AlFeSi}$ ) и парафиновой матрицы, восстановлена магнитная проницаемость сплава. Измерения проницаемостей проведены в коаксиальной линии в диапазоне частот 0.05-20 GHz. Рассмотрено влияние на СВЧ магнитные свойства композитов концентрации, формы и размера частиц порошка. Получено хорошее согласие результатов измерений с формулой Максвелла-Гарнетта, обобщенной с учетом формы частиц, порога протекания и скин-эффекта. Найденные при этом размеры частиц согласуются с данными электронной микроскопии и гранулометрии. И частота, и добротность линии ферромагнитного резонанса для пластинчатых частиц оказываются выше, чем для сферических. Показано, что порошки альсифера являются перспективным наполнителем для радиопоглотителей.

Исследование выполнено при финансовой поддержке Российского научного фонда (проект № 16-19-10490).

DOI: 10.21883/FTT.2017.11.45058.20k

\section{1. Введение}

При проектировании различных высокочастотных (ВЧ) и сверхвысокочастотных (СВЧ) устройств необходимо знать магнитные свойства используемых материалов. В металлы электромагнитное поле проникает лишь на глубину скин-слоя, составляющую на СВЧ доли микрона, поэтому частотные зависимости магнитной проницаемости $\mu$ металлов до настоящего времени оценивались лишь по свойствам тонких пленок.

Известны попытки оценить СВЧ магнитную проницаемость металла по потерям в металлических сетках [1], по зависимости добротности резонатора от внешнего магнитного поля [2], по потерям в коаксиальной линии передачи из исследуемого металла [3]. В этих работах исследовались разные металлы, а полученные оценки $\mu$ относятся к отдельным далеко разнесенным частотным точкам и плохо согласуются друг с другом.

Оценки высокочастотной проницаемости ферромагнетика по измерениям магнитной проницаемости композита, наполненного дисперсными частицами металла (двухфазной механической смеси из порошка наполнителя и полимерного связующего), появились позднее в связи с развитием теории эффективных свойств неоднородных материалов. Так, в работе [4] получена оценка магнитной проницаемости железа из измеренных проницаемостей композитов со сферическими частицами железа. Для этой оценки была использована формула Максвелла-Гарнетта [5]. В результате получено, что получаемая магнитная проницаемость наполнителя зависит от того, композит с какой концентрацией включений был выбран для анализа данных. Очевидно, что этот результат связан с выходом за границы применимости использованной формулы смешения.
В работе [6] собственная магнитная проницаемость частиц альсифера восстановлена из измеренной проницаемости композитов, наполненных частицами этого сплава, с использованием приближения Винера [7], которое применимо лишь при малом контрасте свойств компонентов смеси.

Известны работы $[8,9]$, в которых подобные оценки проведены с применением теории эффективной среды (ТЭС или обобщенной формулы Брюггемана для симметричной смеси [10]). Справедливость применения формулы ТЭС была обоснована в этих работах лишь тем, что измеренные концентрационные зависимости статической магнитной проницаемости композита схожи с кривыми, предсказываемыми выбранной формулой смешения. При этом явная невозможность одновременного описания эффективных материальных параметров композита, диэлектрической проницаемости $\varepsilon_{\operatorname{mix}}$ и магнитной проницаемости $\mu_{\text {mix }}$, при помощи выбранной формулы смешения игнорировалась.

Очевидным недостатком известных попыток определения магнитной проницаемости металла по проницаемости композитов с дисперсными частицами металла является произвольный выбор формулы смешения и определение не свойств частиц, а формальных параметров этой формулы. Поэтому получаемые результаты определяются не столько свойствами металла, сколько видом используемой формулы смешения. В данной работе формула смешения описывает одновременно зависимости $\varepsilon_{\text {mix }}$ и $\mu_{\text {mix }}$ от состава композита, а применимость формулы подтверждена сравнением ее параметров, полученных из эксперимента, с результатами их оценки независимыми методами.

Точный и наиболее корректный подход к связи проницаемостей гетерогенной системы и ее компонентов 
описывается теорией Бергмана-Милтона [11] через характеристическую спектральную функцию, связанную с формой кластеров включений. Определение вида этой функции требует значительных усилий для каждого нового образца смесей, поэтому практического применения использование спектральной функции пока не нашло.

Наиболее близкая к данной работе публикация [12] посвящена выводу гибридной формулы смешения, учитывающей зависимость распределения частиц ферромагнитного металла по форме от их концентрации. Эта формула описывает структурное превращение смеси из матричной в симметричную при достижении порога перколяции. Применимость полученного в [12] выражения ограничена частицами с формой, близкой к сферической.

Ниже проведена оценка частотной зависимости магнитной проницаемости металла на СВЧ по результатам измерений частотных зависимостей эффективных материальных параметров $\left(\varepsilon_{\operatorname{mix}}\right.$ и $\left.\mu_{\text {mix }}\right)$ композитов, наполненных высокодисперсным порошком исследуемого металла. В качестве объекта исследования выбран сплав альсифер $\left(\mathrm{Al}_{0.054} \mathrm{Si}_{0.096} \mathrm{Fe}_{0.85}\right)$, являющийся перспективным наполнителем для СВЧ композитов. Были измерены дисперсионные зависимости материальных параметров матричных смесей на основе парафина с варьируемым содержанием сферических или пластинчатых частиц альсифера известного размера. Зависимости материальных параметров исследуемых композитов от объемной концентрации включений $p$ описаны на каждой частоте с использованием модифицированной формулы Оделевского $[13,14]$, учитывающей форму частиц и изменение структуры смеси при достижении порога перколяции. Формула Оделевского учитывает взаимодействие металлических включений в смеси точнее, чем формула Максвелл-Гарнетта [5].

Применимость формулы Оделевского, связывающей $\varepsilon_{\operatorname{mix}}$ и $\mu_{\text {mix }}$ двухкомпонентной смеси с соответствующими параметрами частиц наполнителя, $\varepsilon_{\text {inlc }}$ и $\mu_{\text {incl }}$, и матрицы, $\varepsilon_{\text {host }}$ и $\mu_{\text {hoct }}=1$, подтверждена отсутствием зависимости определяемых из эксперимента параметров теории (формы включений, определяющей коэффициент деполяризации $N$, и критической концентрации $p_{c}$ включений) от контраста $\mu_{\text {inlc }} / \mu_{\text {host }}$ проницаемостей наполнителя и матрицы. При изменении частоты $f$ магнитная проницаемость включения $\mu_{\text {incl }}$ меняется за счет частотной дисперсии в широких пределах, а полученные величины $N$ и $p_{c}$ остаются постоянными. В случае несоответствия использованной формулы смешения свойствам исследуемых композитов наблюдалось бы либо расхождение расчетной и измеренных зависимостей $\varepsilon_{\text {mix }}(p)$ и $\mu_{\text {mix }}(p)$, либо значительная зависимость параметров формулы смешения от контраста $\mu_{\text {incl }} / \mu_{\text {host }}$ и, соответственно, от частоты.

По измеренному набору значений материальных параметров композита $\mu_{\operatorname{mix}}(f, p)$ и $\varepsilon_{\operatorname{mix}}(f, p)$ для каждой частоты $f$ при различных концентрациях включений $p$ определяются величины $N$ и $p_{c}$. Найденные значения усредняются по частоте, что снижает вклад погрешности измерения материальных параметров смесей. По полученным усредненным величинам $N$ и $p_{c}$ и по измеренным значениям $\mu_{\text {mix }}(f, p)$ рассчитывается частотная зависимость эффективной магнитной проницаемости металлического включения $\mu_{\text {incl }}(f)$. По известной проводимости сплава и размерам включения (оцененным микроскопически и уточненным по коэффициенту деполяризации $N$ ), исключается вклад скин-эффекта в $\mu_{\text {incl }}(f)$ и рассчитывается искомая частотная зависимость магнитной проницаемости металла включения $\mu_{\text {metal }}(f)$. Полученные частотные зависимости $\mu_{\text {metal }}(f)$ для частиц сферической и пластинчатой формы аппроксимируются для оценки параметров ферромагнитного резонанса линией Лоренца. При расчете пластинчатая форма частиц приближается сплюснутым эллипсоидом вращения.

\section{2. Техника эксперимента и полученные результаты}

Используемый для приготовления смесей порошок альсифера получен водным распылением расплава. Измерения гранулометрического состава порошков проводились с помощью лазерного анализатора Analyzette 22. Исходный порошок имеет сферическую форму с наиболее вероятным диаметром частиц $4.2 \mu \mathrm{m}$. Ширина гранулометрической плотности распределения по уровню $50 \%$ равна $2-8 \mu \mathrm{m}$.

Пластинчатые частицы альсифера, электронная микрофотография которых приведена на рис. 1, получены размолом сферических частиц в шаровой мельнице в среде этилового спирта. После размола частицы были рассеяны на ситах; для изготовления смесей была отобрана фракция между ситами с размером ячеек 63 и $40 \mu \mathrm{m}$, при этом наивероятнейший эквивалентный

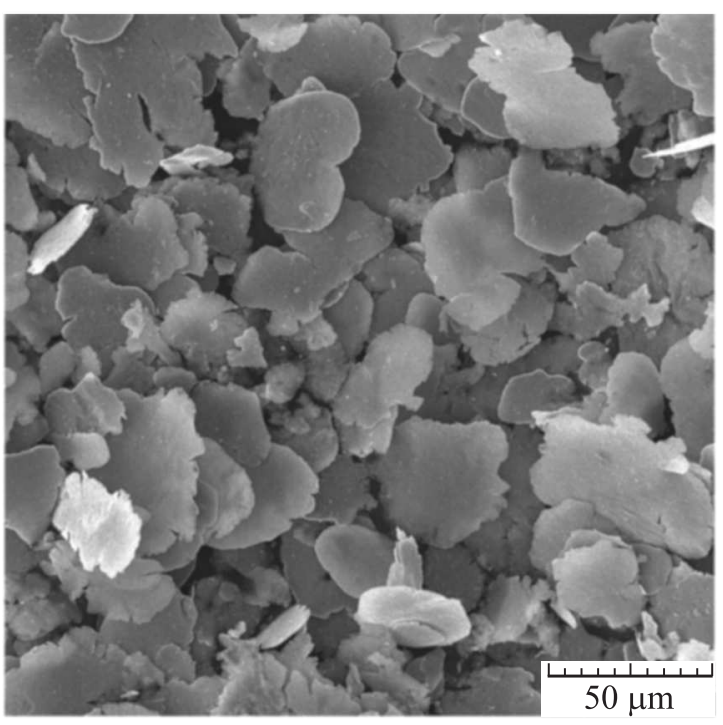

Рис. 1. Электронная микрофотография пластинчатых частиц альсифера, полученных из сферических размолом в шаровой мельнице. 


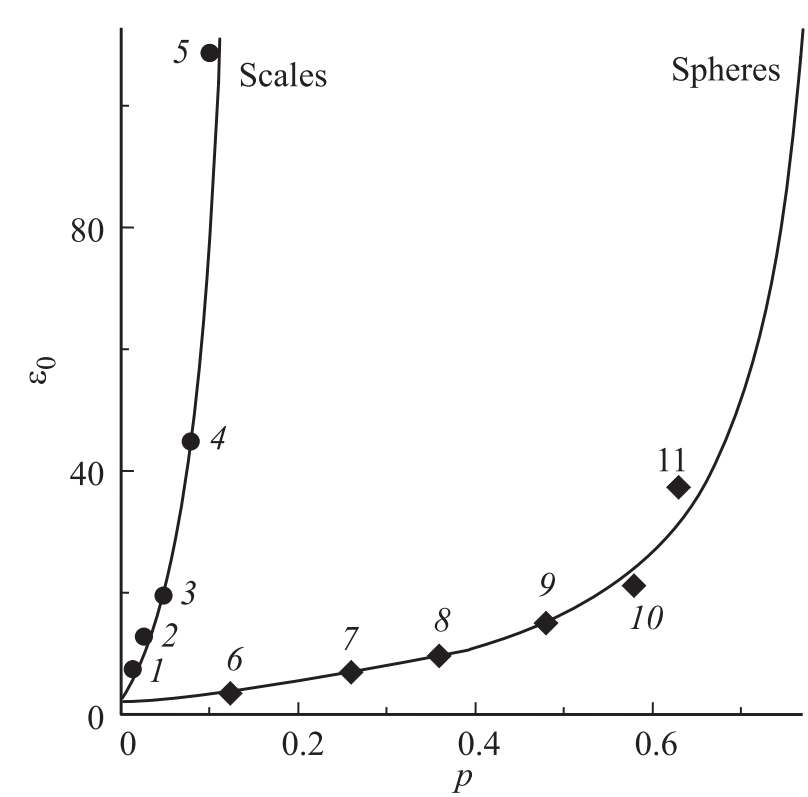

Рис. 2. Зависимость статической диэлектрической проницаемости смесей, наполненных пластинчатыми (кружки 1-5) и сферическими (ромбы 6-11) частицами альсифера от объемной концентрации частиц.

диаметр пластинки составляет около $50 \mu \mathrm{m}$. Толщина частиц, оцененная микроскопически, составляет около $1 \mu \mathrm{m}$.

Композиты для изготовления образцов были изготовлены перемешиванием расчетных навесок порошка альсифера с расплавленным парафином. Перемешивание проводилось при остывании до отверждения парафина. Для улучшения равномерности распределения порошок альсифера смачивался бензином. Полученная после отверждения масса прессовалась в виде шайб (под стандартный размер коаксиальной линии сечением $7 \times 3 \mathrm{~mm})$ толщиной 2-4 mm. Объемная концентрация металла проверялась по плотности шайб; погрешность определения концентрации составляет 0.5-1\%. Максимальное объемное содержание металла в полученных таким образом образцах составляет около 65\% для сферических частиц и около $18 \%$ для пластинчатых частиц.

Для измерения частотной зависимости эффективных материальных параметров шайбы помещались в проходную коаксиальную ячейку и дополнительно прессовались в ней для уменьшения воздушных зазоров между образцом и электродами ячейки, искажающих измеренные значения диэлектрической проницаемости образца. Измерения материальных параметров образцов смесей проводились методом отражения-прохождения [15] в полосе частот $0.05-20 \mathrm{GHz}$ с помощью векторного анализатора цепей Anritsu MS2028.

Диэлектрическая проницаемость образцов смесей во всем рассматриваемом диапазоне частот практически постоянна и может рассматриваться как статическая диэлектрическая проницаемость. Измеренные зависимости диэлектрической проницаемости смесей от объемной концентрации $\varepsilon_{\text {mix }}(p)$ металлических сфер и чешуек представлены на рис. 2.

Измеренная зависимость эффективной магнитной проницаемости композитов от частоты при различных объ-

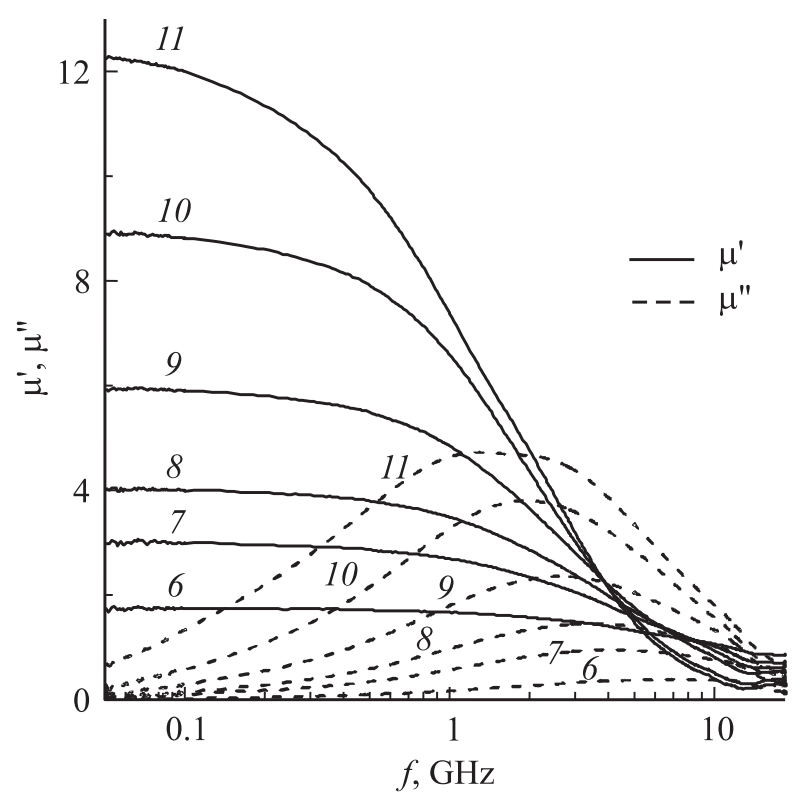

Рис. 3. Зависимость магнитной проницаемости композитов, наполненных сферическими частицами альсифера (действительная часть - сплошные линии, мнимая - пунктирные линии) от частоты. Объемная концентрация сфер равна $p=0.124,0.26,0.36,0.48,0.58,0.63$. (Нумерация кривых соответствует нумерации точек на рис. 2.)

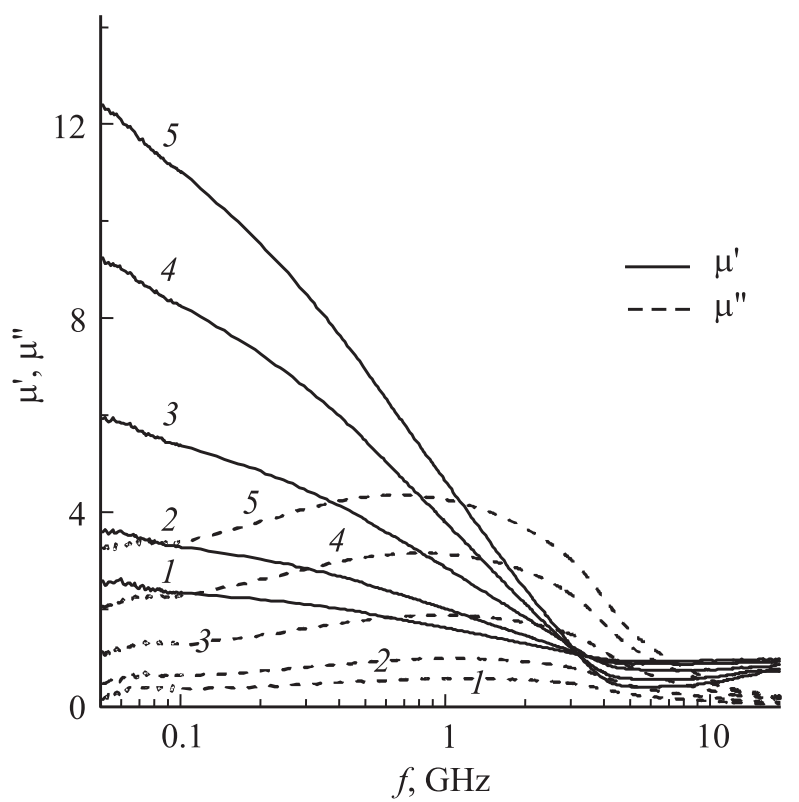

Рис. 4. Зависимость магнитной проницаемости смесей, наполненных пластинчатыми частицами альсифера (действительная часть - сплошные линии, мнимая - пунктирные линии) от частоты. Объемная концентрация чешуек равна $p=0.023$, $0.041,0.077,0.122,0.157$. (Нумерация кривых соответствует нумерации точек на рис. 2.) 
емных концентрациях сферических частиц представлена на рис. 3. Аналогичные данные для случая наполнения композита пластинчатыми частицами приведены на рис. 4.

\section{3. Модифицированная формула Оделевского. Ограничения на величину определяемой проницаемости частиц наполнителя}

Формула Оделевского для матричных смесей описывает широкий круг явлений переноса (обобщенную проводимость [14]) в двухкомпонентных матричных гетерогенных системах (механических смесях). Матричная смесь, по определению [14], состоит из матрицы (непрерывной среды) и наполнителя (отдельных, разделенных прослойками матрицы, включений). В электродинамике формула Оделевского, являющаяся обобщением формулы Максвелл-Гарнетта [5] (в оригинале [5] вывод сделан только для сфер, учет формы включений впервые введен в [14]), используется для описания электрической и магнитной поляризации, или соответственно, диэлектрической $\varepsilon_{\operatorname{mix}}$ (1) и магнитной $\mu_{\operatorname{mix}}$ (2) проницаемости смеси

$$
\begin{gathered}
\varepsilon_{\text {mix }}=\varepsilon_{\text {host }}\left[1+\frac{p}{\left(1-\frac{p}{p_{c}}\right) N+\frac{\varepsilon_{\text {host }}}{\varepsilon_{\text {incl }}-\varepsilon_{\text {host }}}}\right], \\
\mu_{\text {mix }}=1+\frac{p}{\left(1-\frac{p}{p_{c}}\right) N+\frac{1}{\mu_{\text {incl }}-1}} .
\end{gathered}
$$

Формулы (1) и (2) отличаются тем, что диэлектрическая проницаемость композита $\varepsilon_{\text {mix }}$ определяется не только проницаемостью наполнителя $\varepsilon_{\text {incl }}$, но и проницаемостью матрицы, $\varepsilon_{\text {host }}$ (для парафина $\varepsilon_{\text {host }}=2.18$ ). В этих формулах индекс mix относится к смеси, host к матрице, incl - к включениям, $N-$ коэффициент деполяризации (размагничивания), зависящий от формы включения [16], $p$ - объемная доля включений, $p_{c}-$ порог перколяции (критическая концентрация включений, при которой они образуют бесконечный канал протекания - кластер, а структура смеси меняется от матричной к симметричной, в которой оба компонента равнозначны и образуют взаимопроникающие каналы).

Коэффициент деполяризации для сплюснутых эллипсоидов вращения (дисков) при $d / D<1$, где $D-$ больший диаметр, $d$ - толщина, дается формулой [17]

$$
N=\frac{1}{2} \frac{1}{2-2(d / D)^{2}}\left[1-\frac{d / D}{\sqrt{1-(d / D)^{2}}} \operatorname{ArcCos}(d / D)\right] \text {. }
$$

Из (3) следует, что $0<N \leq 1 / 3$.

В расчетах пластинчатые частицы альсифера были аппроксимированы сплюснутыми эллипсоидами вращения, имеющими одинаковую форму. Так как поляризуемость чешуек, стоящих перпендикулярно полю, близка к нулю (коэффициент деполяризации близок к единице $N \rightarrow 1$ ), то эффективная концентрация пластинок в изотропном композите (методика приготовления образцов дает изотропную смесь) составляет $2 / 3$ от концентрации, определяемой по плотности образцов.

В формулах (1), (2) величины $N$ и $p_{c}$ являются параметрами, определяемыми из эксперимента. Теоретически величину коэффициента деполяризации, определяемого только формой включения, можно оценить по выражению (3) из микрофотографий частиц наполнителя, но полученные из них размеры и форма частиц являются лишь оценочными. К тому же, в реальности даже в случае идеально сферических включений в полидисперсной смеси всегда есть кластеры, состоящие хотя бы из двух соседей, что приводит к некоторому уменьшению эффективного коэффициента деполяризации частицы даже в разбавленной смеси по сравнению с теоретической оценкой.

Величина критической концентрации $p_{c}$ является параметром, определяющим взаимодействие между включениями. При $p_{c} \rightarrow \infty$ взаимодействие частиц наполнителя отсутствует. При $p_{c}=1$ учитывается только дипольное взаимодействие между частицами и выражения (1), (2) сводятся к формуле Максвелл-Гарнетта [5]. В реальности $0<p_{c}<1$, причем чем сильнее отличается частица наполнителя от сферы, тем меньше величина $p_{c}$. Заметим, что прямая связь, $p_{c} \approx N$, как в формуле Брюггемана [10], описывающей симметричную смесь, в исследуемых образцах не наблюдается.

Из выражений (1), (2) следуют ограничения на возможность определения проницаемости включений из измеренной проницаемости композита. Так, если проницаемость включений велика, то второе слагаемое в знаменателе формул (1), (2) близко к нулю, и проницаемость смеси определяется только формой и взаимодействием частиц наполнителя. Именно больший контраст, $\left|\varepsilon_{\text {incl }} / \varepsilon_{\text {host }}\right| \sim 10^{9}$, проницаемостей наполнителя и связующего позволяет точнее определять величины $N$ и $p_{c}$ из экспериментальных данных по концентрационной зависимости диэлектрической проницаемости смеси с металлическими включениями $\varepsilon_{\text {mix }}(p)$, чем по концентрационной зависимости магнитной проницаемости. Вследствие большого контраста формула (1) упрощается до (4)

$$
\varepsilon_{\text {mix }} \approx \varepsilon_{\text {host }}\left[1+\frac{p}{\left(1-\frac{p}{p_{c}}\right) N}\right] \text {. }
$$

В этом случае эффективная диэлектрическая проницаемость композита зависит только от трех факторов: от объемной концентрации $p$, от формы включений, описываемой коэффициентом деполяризации $N$, и от взаимодействия включений, описываемого множителем $\left(1-p / p_{c}\right)$. Свойства включения на проницаемость смеси практически не влияют.

При малом контрасте между проницаемостями включения и матрицы, например, на частотах выше частоты ФМР, где $\mu_{\text {incl }} \sim 1$ первое слагаемое знаменателя 
выражений (1), (2) становится много меньше второго, проницаемость смеси, наоборот, перестает зависеть от величин $N$ и $p_{c}$ и, согласно формуле Винера [7], определяется только проницаемостью и концентрацией включений: $\mu_{\text {mix }} \approx 1+p\left(\mu_{\text {incl }}-1\right)$.

Таким образом, анализ концентрационных зависимостей магнитной проницаемости матричных смесей, наполненных магнитными порошками, позволяет определить по проницаемости смеси $\mu_{\text {mix }}$ проницаемость включения $\mu_{\text {incl }}$ лишь в интервале значений, зависящем от формы и концентрации частиц наполнителя. Найдем, дифференцируя выражение (2), влияние коэффициента деполяризации $N$ и концентрации наполнителя $p$ на погрешность определения проницаемости включения $\Delta \mu_{\text {incl }}$ при заданной условиями эксперимента ошибке измерения проницаемости образца $\Delta \mu_{\text {mix }}$

$$
\Delta \mu_{\text {incl }}=\frac{1}{p}\left[\left(\mu_{\text {incl }}-1\right)\left(1-\frac{p}{p_{c}}\right) N+1\right]^{2} \Delta \mu_{\text {mix }} .
$$

Из выражения (5) следует, что ошибка в определении проницаемости включения $\Delta \mu_{\text {incl }}$ пропорциональна разбавлению композита $\left(\Delta \mu_{\text {incl }} \sim 1 / p\right)$, второй степени коэффициента деполяризации $N^{2}$ и эффективной магнитной проницаемости включения $\mu_{\text {incl- }}^{2}$.

Таким образом, определение магнитной проницаемости включений $\mu_{\text {incl }}$ по модели Максвелл-Гарнетта (выражение $(2)$ при $p_{c}=1$ ) имеет удовлетворительную точность лишь в области частот, где величина $\mu_{\text {incl }}$ достаточно низка. Для определения более высоких значений $\mu_{\text {incl }}$ необходимо измерять проницаемость композитов с концентрациями вблизи порога протекания, которые описываются формулой Оделевского (2), точнее учитывающей взаимодействие включений.

При измерениях только магнитной проницаемости $\mu_{\text {mix }}$, из массива экспериментальных данных необходимо определять одновременно три параметра: коэффициент размагничивания $N$, критическую концентрацию $p_{c}$ и искомую проницаемость включения $\mu_{\text {incl. }}$ Такое определение представляет собой сложную экспериментальную задачу, упростить которую можно за счет привлечения дополнительных данных о структуре композита.

В работе [13] предложено определять коэффициент деполяризации $N$ по диэлектрической проницаемости композита $\varepsilon_{\text {mix }}$ (где контраст проницаемостей велик, $\varepsilon_{\text {incl }} / \varepsilon_{\text {host }} \rightarrow 10^{9}$ ) с минимальным содержанием наполнителя. Измерения магнитной проницаемости $\mu_{\text {mix }}$ также проводятся при наполнениях $p \ll p_{c}$, чтобы исключить влияние взаимодействия включений. В методе [13] предъявляются высокие требования к точности измерения $\varepsilon_{\text {mix }}$, так как коэффициент деполяризации $N$ определяется разностью двух близких величин: проницаемости матрицы $\varepsilon_{\text {host }}$ и проницаемости смеси $\varepsilon_{\text {mix }}$ с низким содержанием наполнителя.

В отличие от [13], здесь параметры $N, p_{c}$ и собственно величина проницаемости включения $\mu_{\text {incl }}$ находятся одновременно при помощи минимизации суммы среднеквадратичного расхождения между величинами проницаемостей $\varepsilon_{\operatorname{mix}}(p)$ и $\mu_{\operatorname{mix}}(p, f)$, рассчитанными по формулам (2), (3) и полученными в результате измерения.

При этом обрабатываются экспериментальные данные для одинаковых по форме частиц по всем концентрациям на каждой частоте измерения материальных параметров $\varepsilon_{\text {mix }}(p)$ и $\mu_{\text {mix }}(p)$. В противоположность методу [13], здесь для достоверного определения $N$ и $p_{c}$ необходимы данные по проницаемости композитов в возможно более широком интервале концентраций $p$.

При заданных погрешностях измерения $\Delta \varepsilon_{\text {mix }}$ и $\Delta \mu_{\text {mix }}$ максимальное значение проницаемости включений $\mu_{\text {incl }}$, определяемое предлагаемым методом, ограничивается возможностью достоверно различать величины диэлектрической и магнитной восприимчивостей композита, $\varepsilon_{\text {mix }} / \varepsilon_{\text {host }}-1$ и $\mu_{\text {mix }}-1$. Соответственно, как следует из (5), чем ближе концентрация включений $p$ к порогу перколяции $p_{c}$, тем более высокие величины $\mu_{\text {incl можно }}$ определить.

\section{4. Обработка экспериментальных данных. Расчет магнитной проницаемости частицы наполнителя и составляющего ее металла}

При помощи описанного выше подхода из измеренных значений $\varepsilon_{\text {mix }}(p)$ и $\mu_{\text {mix }}(p, f)$ были определены параметры модели $N$ и $p_{c}$. Величины этих параметров, найденные из данных для различных частот, близки. Полученные отклонения, лежащие в пределах 1-5\%, обусловлены погрешностями измерения материальных параметров композитов. Отсутствие зависимости рассчитываемых параметров от контраста свойств компонентов подтверждает приемлемость выбранной модели смешения.

На рис. 5 черными линиями представлена зависимость магнитной проницаемости $\mu_{\text {incl }}(f)$ сферических частиц альсифера от частоты. Каждая частотная точка на этой зависимости получена при анализе соответствующего массива значений $\varepsilon_{\text {mix }}(p)$ и $\mu_{\text {mix }}(p, f)$, представленных на рис. 2 (кривая сферы) и рис. 3 соответственно.

Аналогично, на рис. 6 черная линия соответствует частотной зависимости магнитной проницаемости $\mu_{\text {incl }}(f)$ пластинчатых частиц альсифера, которая получена обработкой соответствующего массива значений $\varepsilon_{\operatorname{mix}}(p)$ и $\mu_{\text {mix }}(p, f)$, представленных на рис. 2 (кривая диски) и рис. 4 соответственно.

Из сравнения зависимостей $\mu_{\text {incl }}(f)$ для сферических и пластинчатых частиц видно, что на частотах ниже $2 \mathrm{GHz}$ магнитная проницаемость пластинчатых частиц превышает проницаемость сферических, что может быть обусловлено исключением внутреннего объема сферической частицы за счет скин-эффекта (толщина чешуек по данным микроскопии составляет около $1 \mu \mathrm{m}$, а средний диаметр сфер составляет $4.2 \mu \mathrm{m})$. На частоте $100 \mathrm{MHz}$ 


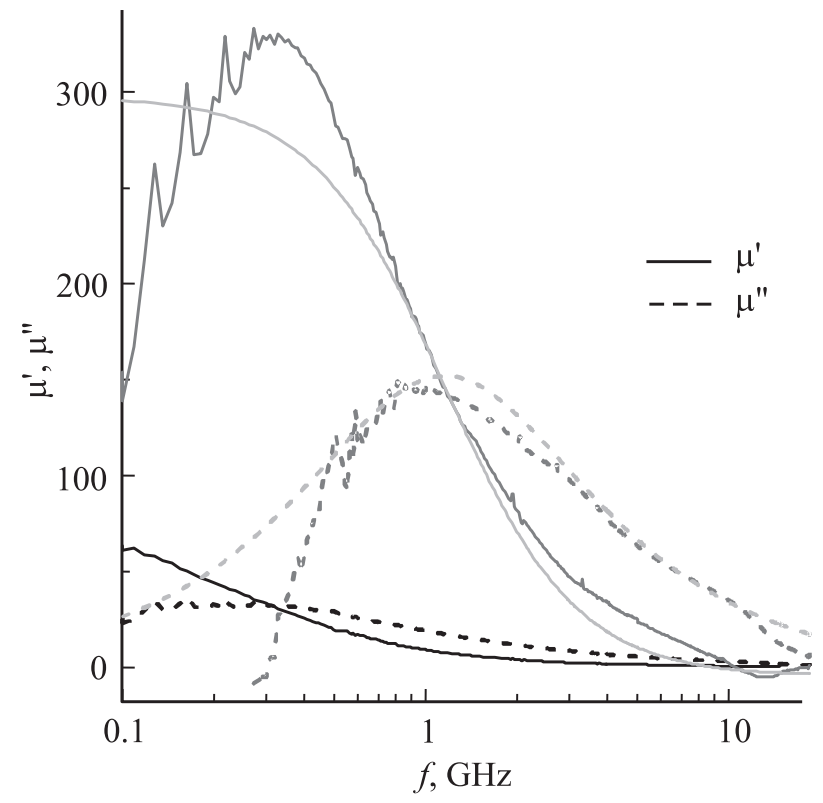

Рис. 5. Зависимость магнитной проницаемости (действительная часть - сплошные линии, мнимая - пунктирные линии) частиц $\mu_{\text {incl }}$ (черные линии) сферической формы и металла частиц $\mu_{\text {metal }}$ (темно-серые линии, в $\mu_{\text {metal }}$ исключен вклад скинэффекта из проницаемости частицы $\left.\mu_{\text {incl }}\right)$ от частоты. Светлосерыми линиями представлена аппроксимация магнитной проницаемости альсифера $\mu_{\text {metal }}$ линией Лоренца.

(нижняя граница экспериментальной полосы частот) магнитная проницаемость обоих включений не выходит на насыщение и далека от статической.

При электропроводности альсифера $\sigma_{\text {incl }} \approx$ $\approx 1.6 \cdot 10^{7} \Omega^{-1} \cdot \mathrm{m}^{-1}$ и толщине (наименьшем размере) $d$ включений можно в каждой частотной точке исключить вклад скин-эффекта из определенной выше магнитной проницаемости включения $\mu_{\text {incl }}(f)$ и рассчитать частотную зависимость магнитной проницаемости сплава, составляющего включение $\mu_{\text {metal }}(f)$. Учет вклада скин-эффекта проводится для сфер и для бесконечной плоскости [17], при этом магнитная проницаемость включения связана с проницаемостью материала включения $\mu_{\text {incl }}=\mu_{\text {metal }} f(\theta)$ через множитель $f(\theta)$, являющийся функцией оптической толщины $\theta$ включения,

$$
\theta=2 \pi f d \sqrt{\varepsilon \mu}
$$

Для плоскости эта функция равна $f(\theta)=\frac{\tan (\theta)}{\theta}$, а для сферического включения

$$
f(\theta)=2 \frac{\tan (\theta)-\theta}{\left(\theta^{2}-1\right) \tan (\theta)+\theta} .
$$

Частотная зависимость магнитной проницаемости альсифера $\mu_{\text {metal }}(f)$ с численно исключенным из $\mu_{\text {incl }}(f)$ вкладом скин-эффекта для сфер представлена на рис. 5 темно-серыми линиями. Аналогично на рис. 6 показана частотная зависимость $\mu_{\text {metal }}(f)$ для пластинчатых частиц. Заметим, что на низких частотах погрешность в толщине пластинки сильно влияет на расчетную величину магнитных потерь: завышение толщины приводит к появлению отрицательных значений мнимой части $\mu_{\text {metal. }}$ Так, толщина пластинчатых частиц, полученная из анализа электронных микрофотографий, близка к $1 \mu \mathrm{m}$, а средний диаметр чешуйки составляет около $50 \mu \mathrm{m}$. При коэффициенте деполяризации $N=0.00854$, найденном как описано выше, отношение толщины эквивалентного эллипсоида (диска) к его диаметру составляет $d / D \approx 0.0011$. Для того, чтобы на частотах ниже $500 \mathrm{MHz}$ мнимая часть магнитной проницаемости $\mu_{\text {metal }}^{\prime \prime}$ была блика к нулю (темно-серая пунктирная линия на рис. 6), толщина чешуйки должна быть около $d \approx 0.65 \mu \mathrm{m}$. Отсюда при $d / D \approx 0.0011$ эквивалентный диаметр чешуйки равен $D \approx 58 \mu \mathrm{m}$, что хорошо согласуется с размером ячеек ситового сепаратора (сторона ячейки $63 \mu \mathrm{m})$.

Заметим, что при обработке экспериментальных данных (рис. 2-4) по формуле Максвелл-Гарнетта, эквивалентный диаметр чешуйки превышает $100 \mu \mathrm{m}$, что значительно больше ячейки сита, а отклонения расчетной проницаемости смеси $\mu_{\text {mix }}(f)$ от измеренной при больших и малых наполнениях превышают $15 \%$.

Наблюдаемое на рис. 5 и 6 на частотах ниже $\sim 300 \mathrm{MHz}$ падение действительной части магнитной проницаемости материала включения $\mu_{\text {metal }}^{\prime}$ с уменьшением частоты обусловлено, по-видимому, ограничениями используемого метода определения магнитной проницаемости металла. С одной стороны, при уменьшении частоты растет погрешность измерения $\mu_{\operatorname{mix}}(f)$

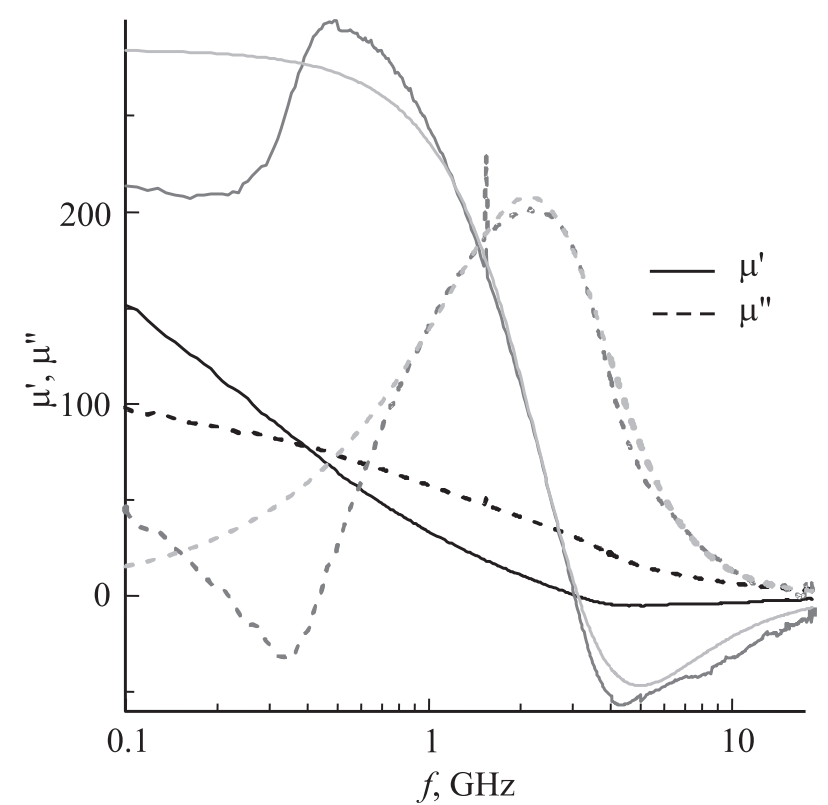

Рис. 6. Зависимость магнитной проницаемости (действительная часть - сплошные линии, мнимая - пунктирные линии) частиц $\mu_{\text {incl }}$ (черные линии) пластинчатой формы и металла частиц $\mu_{\text {metal }}$ (темно-серые линии, в $\mu_{\text {metal }}$ исключен вклад скинэффекта из проницаемости частицы $\mu_{\text {incl }}$ ) от частоты. Светлосерыми линиями представлена аппроксимация магнитной проницаемости альсифера $\mu_{\text {metal }}$ линией Лоренца. 
из-за малой оптической толщины исследуемых образцов. С другой стороны, магнитная проницаемость включений $\mu_{\text {incl }}(f)$ увеличивается из-за частотной дисперсии и уменьшения вклада скин-эффекта и проявляется погрешность, обусловленная неравенством (5). Повысить точность определения магнитной проницаемости металла на частотах ниже $500 \mathrm{MHz}$ можно за счет использования более точных (на частотах мегагерцового диапазона) методов измерения $\mu_{\operatorname{mix}}(f)$, а также за счет изготовления образцов с более высоким содержанием включений. Однако дальнейшее повышение содержания наполнителя при используемой технологии изготовления образцов оказывается проблематичным, так как образцы становятся хрупкими и разрушаются при извлечении из пресс-формы.

Для оценки магнитной проницаемости альсифера на частотах ниже $500 \mathrm{MHz}$ была сделана аппроксимация (7) полученных частотных зависимостей магнитной проницаемости альсифера резонансной кривой (линией Лоренца)

$$
\mu_{\text {metal }}=1+\frac{\mu_{\text {static }}-1}{1-\left(\frac{f}{F}\right)^{2}+i \frac{\Gamma f}{F}} .
$$

В этом выражении $\mu_{\text {static }}$ - статическая магнитная проницаемость металла, $F-$ частота ФМР, $\Gamma-$ коэффициент затухания, $f$ - частота, на которой рассчитывается $\mu_{\text {metal. }}$.

На рис. 5 и 6 рассчитанные частотные зависимости $\mu_{\text {metal }}$ представлены темно-серыми линиями, а аппроксимация - светло-серыми.

Из рисунков видно, что коэффициент затухания для аппроксимирующих лоренцевых функций достаточно высок. Количественное сравнение резонансных частот $F$ при этом проблематично, поэтому приходится рассматривать частоту максимума поглощения. Величины статической магнитной проницаемости альсифера, полученные для сферических и пластинчатых частиц, близки к 300. Максимум поглощения альсифера в пластинчатых частицах находится на частоте около $3 \mathrm{GHz}$, тогда как в сферических частицах он расположен заметно ниже, около $1 \mathrm{GHz}$.

Смещение максимума поглощения альсифера в пластинчатых частицах в область более высоких частот по сравнению с поглощением того же сплава в сферических частицах может быть обусловлено зависимостью условий ФМР от формы частицы. Так, если один размер однородно намагниченного ферромагнетика много меньше других (пленка или плоскость), то частота ФМР будет определяться не только полем анизотропии, но и разностью коэффициентов размагничивания. При этом величина статической проницаемости оказывается близка к величине, наблюдаемой в теле с близкими размерами по всем направлениям (сфере). То есть, параметры частотной зависимости магнитной проницаемости ферромагнетика обусловлены не только его составом, но и формой тела, в котором наблюдается ФМР.

\section{5. Выводы}

Показано, что используемая модель Оделевского хорошо согласуется с измеренными частотными и концентрационными зависимостями материальных параметров композитов. Применимость выражения (2) подтверждена постоянством параметров $N$ и $p_{c}$ при изменении контраста проницаемостей компонентов смеси в широких пределах, а также соответствием эквивалентного диаметра чешуйки $D$, рассчитанного из коэффициента размагничивания $N$ и толщины скин-слоя (6), размерам ячеек ситового сепаратора.

Обработка экспериментальных данных по формуле Максвелл-Гарнетта приводит к выходящему за рамки экспериментальной погрешности расхождению величин расчетной и измеренной проницаемости композита. Кроме того, рассчитанный (при $\left.p_{c}=1\right)$ диаметр чешуйки $D$ превышает размер ячейки сита почти вдвое $(100$ и $63 \mu \mathrm{m}$ соответственно).

Очевидным недостатком матричных моделей является представление композита в виде регулярной структуры, подобной кристаллической решетке. Предполагается, что локальные флуктуации концентрации отсутствуют, а взаимодействие, характеризуемое параметрами $N$ и $p_{c}$, между всеми включениями одинаково. В реальности существует распределение включений по эффективной форме, описываемое характеристической геометрической функцией Бергмана-Милтона $[11,18]$, а слагаемое $\left(1-p / p_{c}\right) N$ в знаменателе формул (3), (4), является лишь уточненным приближением по сравнению с $(1-p) N$ в формуле Максвелл-Гарнетта.

Принятые приближения приводят к незначительным (3-5\%) отклонениям рассчитанной по найденным величинам $N, p_{c}$ и $\mu_{\text {incl }}(f)$ проницаемости смеси $\mu_{\text {mix }}(f)$ от измеренных величин при минимальном $(p \rightarrow 0)$ и максимальном $\left(p \rightarrow p_{c}\right)$ наполнении смесей. Аналогичное незначительное отличие проницаемости, рассчитанной по модели Оделевского, от измеренной, получено при исследовании композитов с порошками железа [13].

Таким образом, формула Оделевского, модифицированная с учетом порога перколяции, точнее описывает свойства матричных смесей, чем формула МаксвеллГарнетта. Найденные величины параметров формулы смешения подтверждаются результатами независимых измерений.

Чем более широкий диапазон наполнений и контраста проницаемостей компонентов смеси описывает выбранная формула смешения, тем меньше погрешность восстановления проницаемости частицы из проницаемости смесей.

По сравнению с обработкой результатов измерения только магнитной проницаемости $\mu_{\text {mix }}(f, p)$, одновременная обработка частотных и концентрационных зависимостей обоих материальных параметров $\varepsilon_{\text {mix }}(f, p)$ и $\mu_{\text {mix }}(f, p)$ смеси позволяет точнее определить параметры формулы смешения, и соответственно, точнее восста- 
новить частотную зависимость $\mu_{\text {metal }}(f)$ проницаемости альсифера в области больших величин $\mu_{\text {metal }}$.

Значительное увеличение погрешности определения $\mu_{\text {metal }}$ на частотах ниже $\sim 300 \mathrm{MHz}$ обусловлено как ростом ошибки в измерении материальных параметров композита методом прохождения-отражения, так и нелинейностью связи (2) проницаемости частицы с проницаемостью смеси. Повышение точности определения $\mu_{\text {metal }}$ на низких частотах возможно при выборе более точного метода измерения материальных параметров и изготовлением образцов с большим содержанием наполнителя.

Повышение точности определения больших величин $\mu_{\text {metal }}$ в принципе возможно за счет уменьшения эффективной магнитной проницаемости включения $\mu_{\text {incl }}$ при наполнении смесей частицами большей толщины $d$. При этом величина $\mu_{\text {incl }}$ будет меньше за счет скин-эффекта, и соответственно будет меньше погрешность ее измерения при той же форме и концентрации включений. Но увеличение точности измерения эффективной проницаемости включения происходит за счет экранирования большей части внутреннего объема частицы, а точный учет вклада скин-эффекта возможен лишь для сферы и бесконечной плоскости. По-видимому, увеличение размера частиц приведет лишь к замене погрешности определения $\mu_{\text {incl }}$ на погрешность учета скин-эффекта.

Измеренные частотные зависимости диэлектрической и магнитной проницаемостей смесей показывают, что исследуемые частицы альсифера могут служить в качестве наполнителя для поглотителей электромагнитного излучения и материалов, обеспечивающих электромагнитную совместимость на частотах ниже $1 \mathrm{GHz}$, превосходящего по уровню магнитных потерь карбонильное железо. В то же время, из восстановленных магнитных спектров сплава $\mathrm{Al}_{0.054} \mathrm{Si}_{0.096} \mathrm{Fe}_{0.85}$ видно, что полученные величины магнитной проницаемости композита $\mu_{\text {mix }}(f)$ ограничены, прежде всего, скин-эффектом и могут быть существенно повышены увеличением дисперсности частиц.

\section{Список литературы}

[1] W. Arkadiew. Ann. Physik 58, 12, (1919).

[2] J.H.E. Griffits. Nature 158, 670 (1946).

[3] G.F. Hodsman, G. Eichholz, R. Millership. Proc. Phys. Soc. Sec. B, 377 (1949).

[4] P. Lederer, C. Brewitt-Taylor. IEEE Proc. SCI. Meas. Techn. 147, 4, 209 (2000).

[5] J.C. Maxwell Garnett. Phil. Trans. R. Soc. Lond. A 203, 385 (1904).

[6] M. Han, D. Liang, K.N. Rozanov, L. Deng. IEEE Trans. Magn. 49, 3, 982 (2013).

[7] O. Wiener. Abh. Math. Phys. Klasse Konigl. 32, 509, (1912).

[8] L.V. Panina, A.S. Antonov, A.K. Sarychev, V.P. Paramonov, E.V. Timasheva, A.N. Lagarkov. J. Appl. Phys. 76, 10, 6365 (1994).

[9] A.N. Lagarkov, V.N. Semenenko, V.A. Chistyaev, I.T. Iakubov. J. Magn. Magn. Mater. 324, 3402 (2012).
[10] D.A. Bruggeman. Ann. Phys., Lpz. 24, 636 (1935).

[11] K.N. Rozanov, M.Y. Koledintseva, J. Drewniak. J. Magn. Magn. Mater. 324, 1063 (2012).

[12] A.V. Osipov, K.N. Rozanov, N.A. Simonov, S.N. Starostenko. J. Phys.: Condens. Matter 14, 9507 (2002).

[13] K.N. Rozanov, A.V. Osipov, D.A. Petrov, S.N. Starostenko, E.P. Yelsukov. J. Magn. Magn. Mater. 321, 738 (2009).

[14] В.И. Оделевский. Расчет обобщенной проводимости гетерогенных систем. Дис. канд. НИИ 34, Ленинград (1946).

[15] A.M. Nicolson, G.F. Ross. IEEE Trans. Instrum. Meas. IM-19, 4, 377 (1970).

[16] J.A. Osborn. Phys. Rev. 67, 11-12, 351 (1945).

[17] Ф. Франк, Р. Мизес. Дифференциальные и интегральные уравнения математической физики. ОНТИ, Ленинград (1937).

[18] K.N. Rozanov, Z.W. Li, L.F. Chen, M.Y. Koledintseva. J. Appl. Phys. 97, 013905 (2005). 